\title{
Assessing follow-up care compliance in children hospitalized for traumatic brain injuries
}

Hannah Bozell BS ${ }^{1}$, Ashley Vetor $\mathrm{MPH}^{2}$, Jodi Raymond $\mathrm{MPH}^{3}$, Alexandra Hochstetler $\mathrm{BS}^{1}$, Teresa M. Bell PhD ${ }^{4 *}$

${ }^{1}$ Indiana University School of Medicine, ${ }^{2}$ Department of Surgery, Riley Hospital for Children at Indiana University Health, , ${ }^{3}$ Trauma Epidemiologist Research Coordinator, Riley Hospital for Children at Indiana University Health, ${ }^{4}$ Department of Surgery, Riley Hospital for Children at Indiana University Health

Background and Hypothesis: There is limited information regarding healthcare utilization and outcomes in children hospitalized for traumatic brain injury (TBI). Nearly $50 \%$ of adults hospitalized for trauma do not attend follow-up appointments, although completion of postdischarge care is associated with improved outcomes and decreased likelihood of subsequent emergency department (ED) visits. The Regestrief Institute Indiana Network for Patient Care (INPC) is a regional health information exchange (HIE) with health record data. This includes inpatient, outpatient, and ED visits, as well as imaging and lab data. The objective of this study is to use HIE data to assess long-term healthcare utilization, complications, and sequelae of pediatric patients hospitalized for TBI to see if follow-up compliance can identify patients at risk for post-TBI complications, including unplanned care, as well as long-term secondary health conditions.

Methods: 387 patients treated at a pediatric level 1 trauma center in Indiana admitted for TBI were identified using trauma registry data. EHR data in the INPC on patients for two years postdischarged were analyzed. Associations between compliance with follow-up care instructions given at discharge/subsequent medical visits and longitudinal utilization/outcomes were examined using Fisher's exact test.

Results: After reviewing patient records, we found that $60.7 \%$ of patients received all follow-up care and $8.5 \%$ of patients received partial follow-up care, leaving $25.1 \%$ of patients receiving no follow up care and $5.7 \%$ of patients lost to follow-up after discharge. $12 \%$ of patients went to the ER for an injury-related issue and $6.2 \%$ of patients were readmitted. $19.4 \%$ of individuals experienced complications from injury while $12.4 \%$ of individuals had suspected sequela. Factors influencing recovery included race, age, insurance, injury severity, ICU admission, and ventilator usage.

Implications and Importance: Using HIE data can identify factors of hospitalized children vulnerable to not achieving optimal recovery and determine what care is critical to improving long-term health and quality of life outcomes. 